\title{
Urgences
}

\section{Sombre jardin de pierres}

\section{Richard Giguère et André Marquis}

Numéro 16, mars 1987

D.G. Jones : d'un texte, d'autres

URI : https://id.erudit.org/iderudit/025388ar

DOI : https://doi.org/10.7202/025388ar

Aller au sommaire du numéro

Éditeur(s)

Urgences

ISSN

0226-9554 (imprimé)

1927-3924 (numérique)

Découvrir la revue

Citer ce document

Giguère, R. \& Marquis, A. (1987). Sombre jardin de pierres. Urgences, (16), 50-51. https://doi.org/10.7202/025388ar d'utilisation que vous pouvez consulter en ligne.

https://apropos.erudit.org/fr/usagers/politique-dutilisation/ 


\section{Richard Giguère/André Marquis SOMBRE JARDIN DE PIERRES}

Sous la pluie, il déploie

ses ombres, tombe

de fleurs séchées

Il revêt les couleurs

des feuilles mortes. Maintenant

ce sont les pierres qui fleurissent

à l'image de ces écrits

qui prolongent le cri

des auteurs disparus

De plus en plus ma bouche

se nourrit de pierres vives

et les os de mes confrères

ressemblent à des fleurs

Que représente ce fouillis, le Jardin des délices

ou le temple d'Angkor Vat

ou le centre-ville après

22 heures? $\mathrm{Ni}$

vivant ni mort

ni humain. Je le traverse

sombre sous la pluie. Le jardin

cultive son alphabet secret 
La plupart de nos expériences de traduction littéraire ont été à la fois des expériences de plaisir de jouer avec les mots et d'insatisfaction, même quelquefois de frustration devant les résultats obtenus. C'est que la traduction est bien un acte de création, une écriture à part entière qui entraîne des incertitudes, des doutes, mais aussi l'exaltation du travail bien fait une fois terminé. Le hic, c'est que justement le travail du traducteur n'est jamais achevé, comme tout travail d'écriture sans doute, mais ici plus qu'ailleurs. Car il faut tenir compte de l'autre texte à l'origine de la traduction, en faisant preuve d'une plus ou moins grande fidélité à ce texte - cela peut se discuter sans fin -, mais le fait est qu'on doit en tenir compte.

Le choix de se mettre à deux pour traduire le poème semé d'embûches de D.G. Jones a eu pour effet d'amplifier le plaisir des découvertes, solitaires ou communes, sans pour autant faire disparaître complètement le sentiment de frustration lié à l'exercice de la traduction: c'est-à-dire essentiellement l'expérience des limites de ce qu'une langue peut transposer d'une autre langue sans dénaturer un texte dans le processus. Dans notre cas, nous avons au cours de nos rencontres noté ces limites, sans nous laisser irriter par elles outre mesure, mais en tentant plutôt de les dépasser ou de les contourner en trouvant des solutions de rechange.

Le texte final a nécessité trois rencontres. La première, qui a duré plus de trois heures, nous a permis de nous entendre sur une transcription littérale du texte, et nous nous sommes amusés à en retracer les multiples écheveaux sonores, sémantiques et rythmiques. Nous avons vite compris qu'il nous serait impossible de rendre les multiples jeux de mots et de conserver certaines sonorités qu'on ne retrouve pas en français. Il fallait dès lors faire des choix! À défaut de respecter le texte original, nous allions créer de nouveaux effets poétiques qui cadreraient mieux avec notre sensibilité et qui mettraient en évidence la lecture que nous voulions privilégier. Notre deuxième rencontre fut plus brève, mais tout aussi fructueuse, puisque nous avons fait des choix déterminants quant à l'interprétation du poème. Ainsi, nous avons supprimé certains mots et nous avons opté pour des transcriptions non littérales. Lors de notre dernière rencontre, nous nous sommes mis d'accord sur une version finale du texte, en gardant à l'esprit que nous pourrions toujours ajouter ceci ou cela au poème. Après plusieurs tentatives, nous parvenions enfin à traduire, de façon satisfaisante, la troisième strophe, qui nous a donné bien du fil à retordre. Est-ce que cette expérience de traduction faite en collaboration, qui fut stimulante pour nous, a donné des résultats probants en termes de produit fini? Aux lecteurs de comparer, d'évaluer, de juger le poème sur ses seuls mérites. 